\title{
Collective excitations and instabilities in double- wire electron - hole systems
}

To cite this article: N Mutluay and B Tanatar 1997 J. Phys.: Condens. Matter 99819

\section{Related content}

- Exchange - correlation effects in
$\frac{\text { semiconductor double-quantum-wire }}{\text { systems }}$
N Mutluay and B Tanatar
- Coupled charged Bose quantum wires
R K Moudgil, K Tankeshwar, K N Pathak
et al.
- Diplons in the degenerate regime:
exchange and correlation effects
B Tanatar

View the article online for updates and enhancements.

\section{Recent citations}

\author{
Mass-asymmetry effects in coupled \\ electron-hole quantum wire system \\ R. K. Moudgil et al \\ Collective charge-density excitations in \\ two-component one-dimensional quantum \\ plasmas: Phase-fluctuation-mode \\ dispersion and spectral weight in \\ semiconductor quantum-wire \\ nanostructures \\ S. Das Sarma and E. H. Hwang
}

\section{IOP ebooks}

Bringing you innovative digital publishing with leading voices to create your essential collection of books in STEM research. Start exploring the collection - download the first chapter of every title for free. 


\title{
Collective excitations and instabilities in double-wire electron-hole systems
}

\author{
N Mutluay and B Tanatar \\ Department of Physics, Bilkent University, Bilkent, 06533 Ankara, Turkey
}

Received 6 May 1997, in final form 12 August 1997

\begin{abstract}
We investigate the collective excitations in a double-wire system of spatially separated electrons and holes. Self-consistent local-field factors are used to describe the exchange-correlation effects at low densities. Plasmon dispersions and the dynamic structure factor $S(q, \omega)$ are studied. Charge-density-wave instabilities in these structures are examined within the linear response theory at small and finite $q$-values.
\end{abstract}

\section{Introduction}

The recent advances in fabrication techniques such as molecular beam epitaxy and metalorganic chemical vapour deposition have made it possible to study the quasi-one-dimensional (Q1D) electron systems in semiconducting structures. Motivated by the technological potential of high-speed electronic devices, experimental and theoretical work in this area continues to be of interest. In this paper we study the collective modes and charge-densitywave instabilities in a double-quantum-wire electron-hole system at zero temperature. These structures, like the double-quantum-well systems that have been recently studied, are important in our understanding of the correlation effects in low dimensions.

The collective excitations in GaAs-based quantum-wire systems have been experimentally studied by spectroscopic methods [1,2]. Theoretical investigations of plasmons [3-7] in Q1D structures have shown that they have a rich excitation spectrum, and that many-body effects are necessary to interpret the experiments. The overall success of the random-phase approximation (RPA) in describing the excitation spectra of quantum wires is attributed $[4,8]$ to the limited phase space of Q1D systems. The validity of using the Fermi-liquid theory (rather than the Tomonaga-Luttinger picture [9]) to describe the semiconducting quantum-wire systems has been discussed by Hu and Sarma [10]. These predictions are in very good agreement with the experimental observation $[1,2]$ of collective excitations in GaAs quantum wires. The ground-state correlation effects in single quantum wires were explored [11], going beyond the RPA. To include corrections due to exchange-correlation effects associated with the charge fluctuations, the method of Singwi et al (STLS) [12] offers an improvement over the RPA. Recently, we employed the STLS method to study the ground-state correlations in double-wire electron and electron-hole systems [13]. In the latter, electrons and holes are confined in separate wires. The self-consistent calculation yields the intrawire and interwire local-field corrections which describe the correlation effects.

Our aim in this paper is firstly to examine the collective modes in double-quantumwire systems, consisting of spatially separated electrons and holes. We specialize to 
equal-density structures, where one wire has electrons as charge carriers and the other one has holes, to study the effects of intrawire and interwire correlations on the plasmon modes. Double-layer electron-hole systems are being fabricated by means of separate electrical contacts [14], and it is conceivable that similar techniques will find application in double-wire structures [15]. Correlation effects are most important when the carrier density $N$ in these systems becomes $\lesssim 10^{5} \mathrm{~cm}^{-1}$. Intrawire and interwire correlations are different in nature because the charge carriers can only move in their respective wires (without tunnelling), and exchange interactions become important. Interwire correlations increase with decreasing wire separation. The STLS approximation has been very useful in increasing our understanding of correlation effects in double-layer two-dimensional electron gas systems [16]. The RPA has been found to overestimate the static properties. On the other hand, the STLS approximation is believed to give reliable results if the carrier density is not too low. In this work we concentrate on the fully self-consistent evaluation of the static structure factors and local-field corrections in electron-hole double-wire systems. Further motivation to study these structures is provided by the electronic instabilities associated with the many-body effects in layered quantum liquids. A charge-density-wave (CDW) instability in double-wire systems has been predicted $[17,18]$ to occur as in the case of double-quantum-well structures [19]. Originating from the many-body interactions, this instability requires an accurate description of the local-field corrections for its analysis.

The rest of this paper is organized as follows. In the next section, we outline the STLS method of calculating the local-field factors self-consistently in a double-wire system, and introduce the quantum-wire model that we use. Section 3 discusses the collective modes in the system. We investigate the CDW instabilities in section 4, and give our conclusions in section 5 .

\section{Theory}

We assume that the Q1D electrons in each wire are embedded in a uniform positive background to maintain the charge neutrality. The linear electron density $n$ is assumed to be the same for the two wires. In terms of the Fermi wave vector we have $n=2 k_{F} / \pi$. The electron gas parameter is defined as $r_{s}=\pi /\left(4 k_{F} a_{B}^{*}\right)$, in which $a_{B}^{*}=\epsilon_{0} /\left(e^{2} m^{*}\right)$ is the effective Bohr radius in the semiconducting wire with background dielectric constant $\epsilon_{0}$ and electron effective mass $m^{*}$. The density-density response function of a double-wire system as extended to a multi-component case [16] involves $\chi_{i i}^{0}(q, \omega)$, the zero-temperature 1D freeelectron density-density response function for the $i$ th wire, and $\psi_{i j}=V_{i j}(q)\left[1-G_{i j}(q)\right]$, the effective interactions ( $i$ and $j$ are the wire indices). We use the particle-number-conserving expression [20]

$$
\chi^{0}(q, \omega ; \gamma)=\frac{(\omega+\mathrm{i} \gamma) \chi^{0}(q, \omega+\mathrm{i} \gamma)}{\omega+\mathrm{i} \gamma\left[\chi^{0}(q, \omega+\mathrm{i} \gamma) / \chi^{0}(q, 0)\right]}
$$

to account for the disorder effects through the phenomenological parameter $\gamma$, in order to justify the use of the Fermi-liquid approach for Q1D electron systems. The fluctuationdissipation theorem enables us to express the static structure factors $S_{i j}(q)$ in terms of the response functions. The $G_{i j}(q)$ are the static local-field factors arising from the shortrange Coulomb interactions and the exchange-correlation effects for the density-density responses. Setting $G_{i j}=0$ in the full density-response function one recovers the usual RPA, which neglects correlation. The intralayer and interlayer local-field factors $G_{i j}(q)$ are obtained within the STLS approach $[11,12]$. The details of the theoretical framework and our self-consistent calculations are presented elsewhere [13]. The model that we use 
for the Q1D electron system is that developed by Gold and Ghazali [6]. It consists of two cylindrical quantum wires of radius $R$ each in an infinite potential well and separated by a distance $d(d>2 R)$. We assume that only the lowest subband in each given quantum wire is occupied. This will be fulfilled for $r_{s}>\left(R / a_{B}^{*}\right) / 4$, especially in the low-density regime in which the many-body effects are important $[6,11]$. The intrawire and interwire Coulomb interactions between the particles are given by Gold and Ghazali [6] and Gold [17], respectively. The main features of this model are that the intrawire potential behaves as $\sim|\ln (q R)|$ for long wavelengths and the interwire potential as $\sim|\ln (q d)|$, characteristic of various other proposals $[3,4]$. The quantum-wire model used for a Q1D structure determines some asymptotic properties of the local-field factor, as discussed by Fantoni and Tosi [21].

We solve the set of equations relating the structure factors and local-field corrections for density-density response in an electron-hole double-wire system self-consistently. The material parameters for GaAs are used, so the electron and hole masses are $m^{*}=0.07 m_{e}$ and $m_{h}^{*}=0.5 m_{e}$ ( $m_{e}$ is the free-electron mass), respectively, and the dielectric constant is $\epsilon_{0}=12.9$. The phenomenological disorder parameter $\gamma$ that enters into the self-consistent calculation does not influence the converged $S_{i j}(q)$ and $G_{i j}(q)$ significantly for $\gamma \lesssim 0.1 E_{F}$. The particle-number-conserving expression that we employ has the same form as in more sophisticated approaches [22], where it is replaced by the wave-vector- and frequencydependent memory function $\gamma(q, \omega)$. The phenomenological $\gamma$ may be related to the measured mobilities in quantum wires by the relaxation-time expression.

\section{Collective modes}

The collective excitations in a double-wire system when correlation effects are included are obtained from the zeros of the screening function

$$
\begin{gathered}
\varepsilon(q, \omega)=\left[1-V_{11}(q)\left[1-G_{11}(q)\right] \chi_{11}^{0}(q, \omega)\right]\left[1-V_{22}(q)\left[1-G_{22}(q)\right] \chi_{22}^{0}(q, \omega)\right] \\
-\left[V_{12}(q)\left[1-G_{12}(q)\right]\right]^{2} \chi_{11}^{0}(q, \omega) \chi_{22}^{0}(q, \omega)=0
\end{gathered}
$$

in which we use the disorder-free response functions. In the case of an equal-density double-wire electron system, the plasmon dispersions are obtained analytically [4, 23], and the ensuing modes are called optical and acoustic plasmons-referring to the in- and out-ofphase oscillations of the charges within the wires. For electron-hole double wires the full collective modes have to be solved numerically. The $q \rightarrow 0$ limit of the dispersion relations may be calculated as for the 2D and Q1D two-component electron liquid cases [24]. The main difference here is that the electron and hole wires are spatially separated. For a given wire radius, when the density is low enough, both modes are above the single-particle continuum of electrons (light particles). We obtain the plasmon dispersions as $(q \rightarrow 0)$

$$
\left[\omega_{\mathrm{pl}}^{\mathrm{op}}(q)\right]^{2}=B / 2+\left(B^{2} / 4-C\right)^{1 / 2}
$$

where

$$
\begin{aligned}
& B=\frac{16 r_{s}}{\pi^{2}} q^{2}\left[F_{11}\left(1-G_{11}\right)+\rho F_{11}\left(1-G_{22}\right)\right] \\
& C=\left(\frac{16 r_{s}}{\pi^{2}}\right)^{2} \rho q^{4}\left[F_{11}^{2}\left(1-G_{11}\right)\left(1-G_{22}\right)-F_{12}^{2}\left(1-G_{12}\right)^{2}\right] .
\end{aligned}
$$

In the above expressions, we measure the plasmon energy in terms of the Fermi energy $E_{F}=k_{F} / 2 m^{*}$ of the electrons, $\rho=m^{*} / m_{h}^{*}$, and $V_{11}(q)=e^{2} F_{11} /\left(2 \epsilon_{0}\right)$, etc. Since the mass ratio $1 / \rho \ll 1$, equation (2) admits another solution (an acoustic plasmon) for energies above the single-particle continuum of holes, and below the single-particle continuum of 
electrons. This happens when the density of the wires is sufficiently high. We find the long-wavelength dispersion of acoustic plasmons to be

$$
\left[\omega_{\mathrm{pl}}^{\mathrm{ac}}(q)\right]^{2}=\frac{\omega_{+}^{2} \mathrm{e}^{A^{\prime} / B^{\prime}}-\omega_{-}^{2}}{\mathrm{e}^{A^{\prime} / B^{\prime}}-1}
$$

where

$$
\begin{aligned}
& A^{\prime}=1-F_{11}\left(1-G_{11}\right)\left(\frac{2 r_{s}}{\pi^{2}}\right) \frac{2}{q} \ln \left|\frac{\omega_{-}}{\omega_{+}}\right| \\
& B^{\prime}=F_{11}\left(1-G_{22}\right)\left(\frac{2 r_{s}}{\pi^{2}}\right) \frac{1}{\rho q}-\left[F_{11}^{2}\left(1-G_{11}\right)\left(1-G_{22}\right)-F_{12}^{2}\left(1-G_{12}\right)^{2}\right] \\
& \times\left(\frac{2 r_{s}}{\pi^{2}}\right)^{2} \frac{2}{\rho q^{2}} \ln \left|\frac{\omega_{-}}{\omega_{+}}\right| .
\end{aligned}
$$
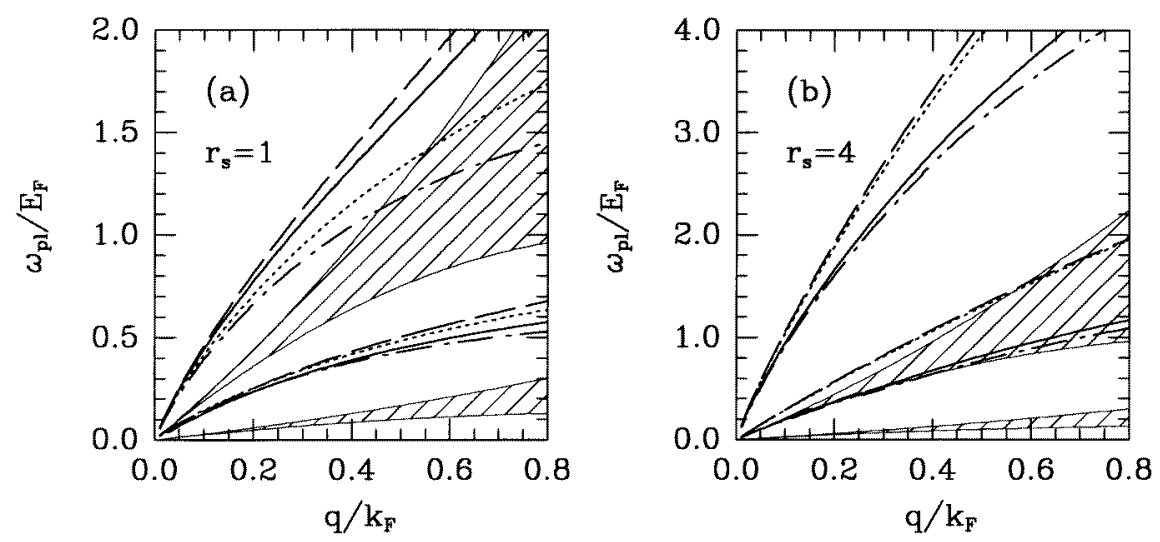

Figure 1. (a) The collective modes in a double-wire electron-hole system for $R=2 a_{B}^{*}$, $d=5 a_{B}^{*}$, and $r_{s}=1$. The solid and dashed lines are the full dispersion curves with and without the local-field effects, respectively. The chain and dotted curves are the corresponding long-wavelength approximations. (b) As (a), but for $r_{s}=4$.

We illustrate the collective modes in a double-wire electron-hole system in figure 1. The optical and acoustic plasmon modes of an $R=2 a_{B}^{*}$ and $d=5 a_{B}^{*}$ system at $r_{s}=1$, are depicted in figure 1(a). The optical plasmons exist above the upper boundary of the particle-hole $(\mathrm{p}-\mathrm{h})$ continuum of electrons, as expected. The solid and dashed lines indicate the full numerical solution of $\operatorname{Re}[\varepsilon(q, \omega)]=0$, with and without the local-field corrections. They correspond to the STLS approximation and the RPA, respectively. The chain and dotted lines are the long-wavelength limits of the full plasmon dispersions with and without the local-field effects, respectively. In general the presence of exchange-correlation effects, described by the static local-field corrections $G_{i j}(q)$, tend to move the plasmon dispersions down from their RPA values. Such behaviour is observed in higher dimensions as well, and stems from the reduced carrier-carrier interaction strength in highly correlated systems. We note that the $q \rightarrow 0$ approximations start to deviate from the full plasmon dispersions at around $q \approx 0.2 k_{F}$, and consequently they enter the particle-hole continuum at some critical wave vector $q_{c}$. The acoustic plasmons are in the region above the $\mathrm{p}-\mathrm{h}$ continuum of holes and below the $\mathrm{p}-\mathrm{h}$ continuum of electrons. The effects of local-field corrections on the acoustic plasmons are similar to those on optical plasmons. However, we observe 
that the $q \rightarrow 0$ approximation in this case describes the full plasmon dispersions better for the range of $q$-values of interest. We now study the collective modes in a lower-density system. Figure 1(b) shows the optical and acoustic plasmon dispersions calculated using the numerical solution of $\operatorname{Re}[\varepsilon(q, \omega)]=0$, and the above long-wavelength expressions for an electron-hole double-wire system at $r_{s}=4$. The full RPA and STLS results are plotted as the dashed and solid lines, respectively. The long-wavelength expressions with and without the local-field corrections are denoted by the chain and dotted lines, respectively. Correlation effects become more important at $r_{s}=4$, and we observe a marked difference between the RPA and STLS approaches. It is also interesting to note that the $q \rightarrow 0$ approximations describe the full dispersion curves better in this situation. Within the RPA, the acoustic plasmons are pushed above the $\mathrm{p}-\mathrm{h}$ continuum of electrons. Similar behaviour of the collective modes is also seen in double-layer systems. On the other hand, at $r_{s}=4$ the exchange-correlation effects have a great influence on the acoustic plasmons. In the STLS approximation, the acoustic plasmon dispersion curve (solid line) is just below the lower boundary of the $\mathrm{p}-\mathrm{h}$ continuum of electrons for small $q$, and it enters the damping region at around $q \approx 0.3 k_{F}$.

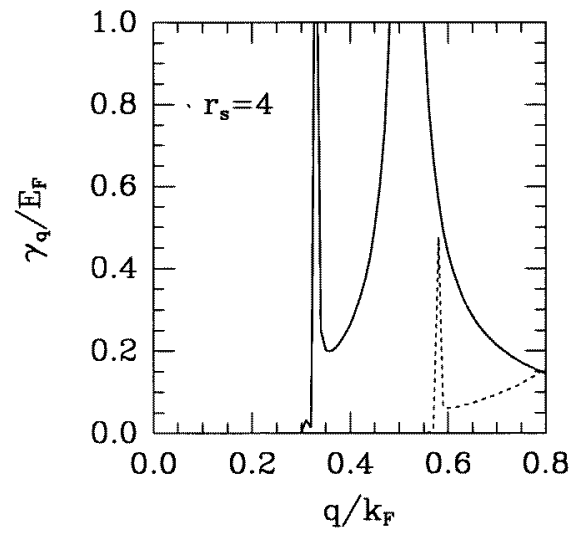

Figure 2. The acoustic plasmon damping rate in a doublewire electron-hole system at $R=2 a_{B}^{*}, d=5 a_{B}^{*}$, and $r_{s}=4$. The solid and dotted lines denote the STLS and RPA results, respectively.

To see this effect from another point of view, we plot in figure 2 the damping rate of acoustic plasmons using

$$
\gamma_{q}=\left|\frac{\operatorname{Im}[\varepsilon(q, \omega)]}{\partial \operatorname{Re}[\varepsilon(q, \omega)] / \partial \omega}\right|_{\omega_{\mathrm{pl}}^{\mathrm{ac}}(q)} .
$$

The solid and dotted lines in figure 2 denote $\gamma_{q}$ in the STLS approximation and the RPA, respectively. The damping $\gamma_{q}$ describes the rate at which the acoustic plasmons decay into single-particle-hole pairs.

The collective modes of a double-wire electron-hole system can also be studied via the dynamic structure factor $S(q, \omega)$, defined as

$$
S(q, \omega)=-\frac{1}{n \pi} \sum_{i j} \operatorname{Im}\left[\chi_{i j}(q, \omega)\right] .
$$

The dynamic structure factor gives a measure of the density fluctuation spectrum and is observed in inelastic electron spectroscopy or Raman scattering experiments. In figure 3 we show $S(q, \omega)$ for an electron-hole double-wire system with $R=2 a_{B}^{*}$ and $d=5 a_{B}^{*}$, at $q=0.2 k_{F}$. We choose the linear carrier density such that $r_{s}=1$. In the RPA the dynamic structure factor (dotted line) exhibits contributions from the particle-hole continua 


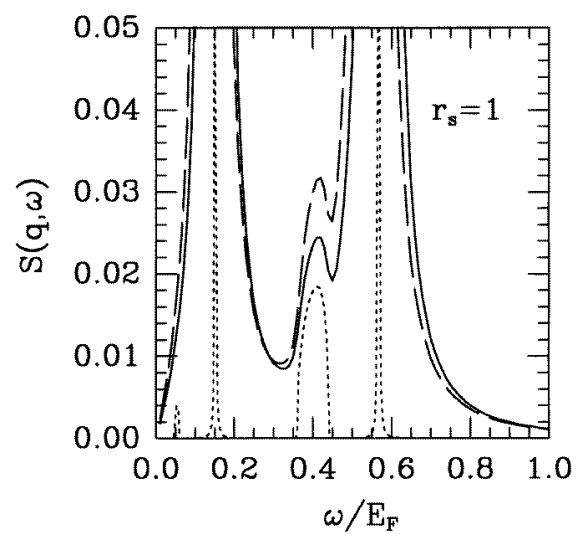

Figure 3. The total dynamic structure factor $S(q, \omega)$ for a double-wire electron-hole system, with $R=2 a_{B}^{*}, d=5 a_{B}^{*}, q=0.2 k_{F}$, and $r_{s}=1$. The dotted, dashed, and solid lines represent the RPA $(\gamma=0)$, the RPA $\left(\gamma=0.01 E_{F}\right)$, and the STLS approximation $\left(\gamma=0.01 E_{F}\right)$, respectively.

of electrons and holes, as well as very sharp plasmon peaks. When we introduce a damping parameter of $\gamma=0.01 E_{F}$, we observe that these peaks are broadened considerably (solid line). The plasmon contributions dominate $S(q, \omega)$. The effect of the local-field factors $G_{i j}(q)$ is to shift the peak positions to the low-energy side (dashed line). This is visible even for the $r_{s}=1$ case of figure 3 . We further remark that as the separation distance between the wires is increased, the acoustic plasmon peak (located at lower energies) decreases in magnitude, whereas the optical plasmon peak increases. Similar behaviours of plasmons in double-layer electron systems were examined by Cordes and Das [25] who also argued for the prospects of experimentally observing the collective modes.

\section{Charge-density-wave instabilities}

In order to study the instabilities in double-wire systems, we look for the divergences in the response function [19]

$$
\chi_{-}(q)=\frac{2}{1 / \chi_{1}(q)+1 / \chi_{2}(q)-\left[\left(1 / \chi_{1}(q)-1 / \chi_{2}(q)\right)^{2}+4 \psi_{12}(q)\right]^{1 / 2}}
$$

where $1 / \chi_{i}(q)=1 / \chi_{i i}^{0}(q)+\psi_{i i}(q)$ is the response function of a single (isolated) wire and $\psi_{i j}(q)=V_{i j}(q)\left[1-G_{i j}(q)\right]$ is the effective interaction between the carriers in wires $i$ and $j$, including the local-field corrections. The condition for a CDW instability corresponds to $1 / \chi_{-}(q \rightarrow 0)=0$. Taking the small- $q$ limit in equation (7) we obtain

$$
\alpha_{c} / R=\exp \left\{\frac{F_{11}\left(k_{F}\right)}{8}-\frac{73}{120}-(1+\rho) \frac{\pi^{2}}{32 r_{s}}\right\}-2>0 .
$$

The approximations leading to the above stability condition are such that for small $q$, we neglect the interwire correlation effects, $G_{12}(q)=0$, and replace the intrawire local-field factors $G_{11}(q)$ and $G_{22}(q)$ by the Hubbard approximation, $G_{H}(q)$. As $q \rightarrow 0$, we use $G_{H}(q) \approx \frac{1}{2} F_{11}\left(k_{F}\right) / F_{11}(q)$. Since the interwire local-field factor $G_{12}$ is appreciable only for large $q$, and the Hubbard approximation describes the long-wavelength limit of the intrawire local fields quite well, the stability condition given in equation (8) provides a 


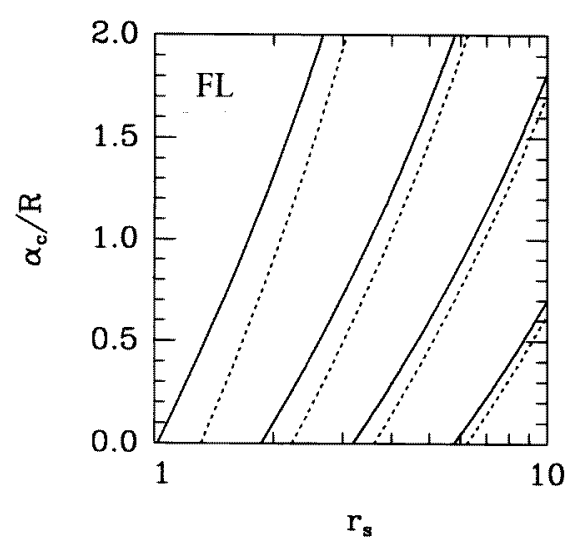

Figure 4. The critical distance $\alpha_{c}=d-2 R$ below which the Q1D Fermi liquid becomes unstable, as a function of $r_{s}$. The solid and dotted lines refer to electron-hole and electron double wires, respectively. The curves from left to right indicate $R=0.1 a_{B}^{*}, 0.5 a_{B}^{*}, 0.75 a_{B}^{*}$, and $a_{B}^{*}$, respectively.

reasonable estimate. Note also that the $r_{s}$-dependence of $G_{H}(q)$ is embodied in $F_{11}\left(k_{F}\right)$. For a given density (or $r_{s}$ ) and wire radius $R$, we conclude that the Fermi liquid is stable for $\alpha_{c}>0$. The critical distance $\alpha_{c}$ as a function of the density parameter $r_{s}$ in an electron-hole double-wire system is shown in figure 4, for various wire radii. For comparison we also show the corresponding case for an electron system (dotted lines) studied by Gold [17]. The electron-hole system is a stable Fermi liquid in the region $\alpha_{c}\left(R, r_{s}\right)>0$ indicated by FL in figure 4. It is observed that the instability in an electron-hole system occurs at smaller $r_{s}$-values (higher densities) than that in an electron system for the same interwire separation distance and wire radius. This is more notable for small $R$, and as the wire radius increases the critical distances $\alpha_{c}$ become essentially the same for electron-hole and electron systems. To see how well the phase diagram given in figure 4 describes the instabilities, we now calculate $\chi_{-}(q)$.

In figure 5 we display $\chi_{-}(q)$ as a function of $q$ (as $q \rightarrow 0$ ) for $R=a_{B}^{*} / 2$ and $r_{s}=5$. As the interwire distance $\alpha$ approaches the critical value a strong peak in $\chi_{-}(q)$ develops around $q \approx 0.03 k_{F}$, validating our previous analysis. We have found that the peak splits into two at a spacing of $\alpha / R \approx 0.55$, whereas similar calculations [18] for double-wire electron systems reported no such splitting. We note that in figure 5 the static susceptibility $\chi_{-}(q)$ is calculated with the local-field corrections $G_{i j}(q)$ from a self-consistent STLS scheme, but their effects are rather small. Similar instabilities in electron double-wire systems were detected by Wang and Ruden [18].

Owing to the perfect nesting of the Fermi surface in 1D, an intrinsic instability occurs at $q=2 k_{F}$, reflected by a singularity in the noninteracting susceptibility $\chi^{0}(q)$. Although thermal fluctuations and collisional broadening effects (characterized phenomenologically by the parameter $\gamma$ ) remove this singularity [26], a peak structure at $2 k_{F}$ persists. We now explore the effects of strong interwire and intrawire interactions on $\chi_{-}(q)$ at finite $q$. In this case, the local-field effects are expected to be important especially at large $r_{s}$. Figure 6 shows $\chi_{-}(q)$ as a function of $q$ within the STLS approximation. At $r_{s}=4$, exchangecorrelation effects become important and a peak structure is observed around $q \approx 0.3 k_{F}$, for various separation distances. Wang and Ruden [18] found divergent behaviour in $\chi(q)$ at $2 k_{F}$ when the wire densities become sufficiently low and the separation distance becomes 


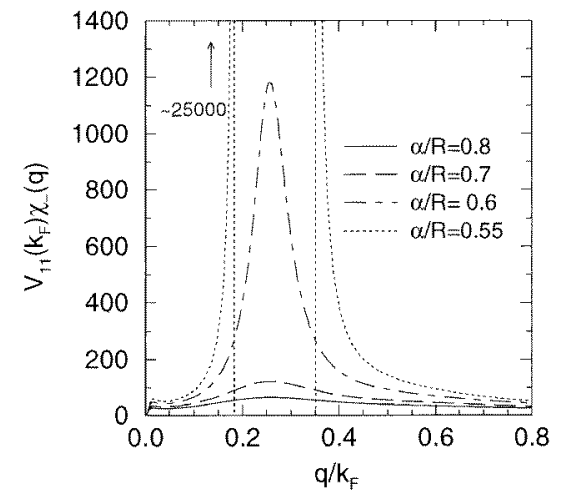

Figure 5. The small- $q$ behaviour of the static response function $\chi_{-}(q)$, for a double-wire electron-hole system at $R=a_{B}^{*} / 2$, and $r_{s}=5$.

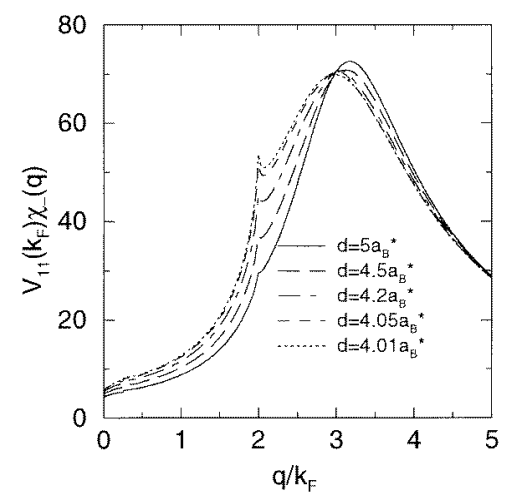

Figure 6. The large- $q$ behaviour of the static response function $\chi_{-}(q)$, for a double-wire electron-hole system at $R=2 a_{B}^{*}$ and $r_{s}=4$ within the STLS approximation.

sufficiently small. We observe similar behaviour in electron-hole double wires, except that the $2 k_{F}$-divergence is substantially reduced because of the phenomenological parameter $\gamma$ (taken typically to be $\gamma \approx 0.01 E_{F}$ ). For double-layer electron-hole systems, Szymański et al [16] have found divergences in the static response at large $r_{s}$ and at a critical spacing between the layers. These divergences were attributed to the onset of charge-density-wave instabilities. For double-wire electron systems, Wang and Ruden [18] argued that the $q \rightarrow 0$ instabilities could not be reached since they occur at smaller wire separations than those at $q=2 k_{F}$. In our calculations, $\chi_{-}(q)$ for double-wire electron-hole systems seems to exhibit a large peak beyond $q=2 k_{F}$ at wire separations comparable to those of the $q \rightarrow 0$ case. Such finite- $q$ instabilities may be a precursor to a fluid-solid phase transition, i.e. Wigner crystallization, as suggested by Shulz [27], which may be detected experimentally.

\section{Concluding remarks}

In this work we have mainly considered equal-density double-wire systems. Our method can easily be generalized to unequal-density situations. It is expected that the collective modes of an unequal-density system will have qualitatively different properties from the modes in identical wires. Liu et al [16] have studied the behaviour of plasmons in double-layer systems with unequal densities. The semiconducting quantum wires realized so far and used in the experiments are typically characterized by densities of $r_{s} \sim 1$. It is conceivable that low-density structures will be manufactured in the future, in view of the advances in growth technology. The many-body effects discussed here would then be more readily applicable to the experimental realizations. The collective modes (plasmons) described in this work can in principle be detected by light scattering experiments similar to the ones performed before $[1,2]$. There is also a possibility of observing the effects of optical and acoustic plasmons in transport measurements, namely in the so-called Coulomb drag effect as demonstrated by Hill et al [28] for 2D systems. Although the actual realization of spatially separated electron-hole wires appears to be quite difficult, we believe that the advances in the growth technology will surmount these difficulties. A host of interesting effects predicted theoretically [29] for these systems show the timeliness of concentrated efforts in this direction. CDW-type instabilities discussed in the context of double-quantum-well structures $[19,23]$ and also in double quantum wires $[17,18]$ could be explored. In summary, we 
have studied the ground-state correlations in Q1D electron-hole systems in double-quantumwire structures interacting via the Coulomb potential within a self-consistent scheme. The local-field corrections describing exchange-correlation effects provide an improvement over the RPA results. We found that the double-wire Q1D electron system, such as occurs in semiconducting quantum wires, shows qualitatively similar behaviour to that found in $2 \mathrm{D}$ and $3 \mathrm{D}$ cases. The collective modes in double-wire systems, particularly in electron-hole wires, exhibit a rich structure which could be probed in Raman-scattering-type experiments.

\section{Acknowledgment}

This work was partially supported by the Scientific and Technical Research Council of Turkey (TUBITAK) under Grant No TBAG-AY/123.

\section{References}

[1] Hansen W, Horst M, Kotthaus J P, Merkt U, Sikorkski C and Ploog K 1987 Phys. Rev. Lett. 582586 Demel T, Heitmann D, Grambow P and Ploog K 1988 Phys. Rev. B 3812732

Goñi A R, Pinczuk A, Weiner J S, Calleja J M, Dennis B S, Pfeiffer L N and West K W 1991 Phys. Rev. Lett. 673298

Schmeller A, Goñi A R, Pinczuk A, Reiner J S, Calleja J M, Dennis B S, Pfeiffer L N and West K W 1994 Phys. Rev. B 4914778

[2] Perez F, Jusserand B, Dahl C, Filoche M, Ferlazzo-Manin L and Etienne B 1996 Phys. Rev. B 54

Dahl C, Jusserand B and Etienne B 1995 Phys. Rev. B 5117211

Dahl C, Jusserand B and Etienne B 1996 Solid State Electron. 40261

[3] Hu G Y and O’Connell R F 1990 Phys. Rev. B 421290

[4] Li Q P and Das Sarma S 1991 Phys. Rev. B 4311768

[5] Hu B Y-K and Das Sarma S 1992 Phys. Rev. Lett. 681750

Hu B Y-K and Das Sarma S 1993 Phys. Rev. B 485469

[6] Gold A and Ghazali A 1990 Phys. Rev. B 417626

[7] Yu J-X and Xia J-B 1996 Solid State Commun. 98227

[8] Das Sarma S and Hwang E H 1996 Phys. Rev. B 541936

[9] Sólyom J 1979 Adv. Phys. 28201

For a fairly recent review, see, e.g.,

Mattis D C (ed) 1993 The Many-Body Problem (Singapore: World Scientific)

[10] See reference [5] for a detailed account of a Fermi-liquid and Luttinger-liquid comparison.

[11] Friesen W I and Bergersen B 1980 J. Phys. C: Solid State Phys. 136627

Campos V B, Degani M H and Hipólito O 1995 Superlatt. Microstruct. 1785

Calmels L and Gold A 1995 Phys. Rev. B 518426

[12] Singwi K S, Tosi M P, Land R H and Sjölander A 1968 Phys. Rev. 179589

Singwi K S and Tosi M P 1981 Solid State Physics vol 36 (New York: Academic) p 177

[13] Mutluay N and Tanatar B 1997 Phys. Rev. B 556697

Mutluay N and Tanatar B 1977 J. Phys.: Condens. Matter 93033

[14] Sivan U, Solomon P M and Shtrikman H 1992 Phys. Rev. Lett. 681196

[15] Komori K, Wang X-L, Ogura M, Matsuhata H and Imanishi H 1996 Appl. Phys. Lett. 683787

Simhony S, Kapon E, Colas E, Bhat R, Stoffel N G and Hwang D M 1990 IEEE Photon. Technol. Lett. 2 305

[16] Zheng L and MacDonald A H 1994 Phys. Rev. B 495522

Liu L, Świerkowski L, Neilson D and Szymański J 1996 Phys. Rev. B 537923

Szymánski J, Świerkowski L and Neilson D 1994 Phys. Rev. B 5011002

Zhang C and Tzoar N 1988 Phys. Rev. B 385786

[17] Gold A 1992 Phil. Mag. Lett. 66163

[18] Wang R and Ruden P P 1995 Phys. Rev. B 527826

[19] Świerkowski L, Neilson D and Szymański J 1991 Phys. Rev. Lett. 67240 Neilson D, Świerkowski L, Szymánski J and Liu L 1993 Phys. Rev. Lett. 714035

[20] Mermin N D 1970 Phys. Rev. B 12362 
Das A K 1975 J. Phys. F: Met. Phys. 52035

[21] Fantoni R and Tosi M P 1996 Physica B 21735

[22] Sjölander A and Stott J 1972 Phys. Rev. B 52109

[23] Gold A 1993 Phys. Rev. B 476762

Gold A 1995 Z. Phys. B 97119

[24] Vignale G 1988 Phys. Rev. B 38811

Tanatar B 1994 Solid State Commun. 92699

Zheng L and Das Sarma S 1996 Phys. Rev. B 5413908

[25] Cordes J G and Das A K 1991 Solid State Commun. 80145

Cordes J G and Das A K 1997 Superlatt. Microstruct. 21195

[26] Das Sarma S and Lai Wu-yan 1985 Phys. Rev. B 321401

[27] Shulz T 1993 Phys. Rev. Lett. 711864

[28] Hill N P R, Nicholls J T, Linfield E H, Pepper M, Ritchie D A, Jones G A C, Hu B Y-K and Flensberg K 1997 Phys. Rev. Lett. 782204

[29] See for instance Sun Y and Kirczenow G 1994 Phys. Rev. Lett. 722450 